\title{
Cure monitoring of a UV cured epoxy resin using a long period grating Mach-Zehnder interferometer
}

\author{
Stephen J. Buggy, Richard P. Murphy, Stephen W. James and Ralph P. Tatam \\ Engineering Photonics Group, School of Engineering, Cranfield University, Bedford, UK
}

\begin{abstract}
A cascaded long period grating Mach-Zehnder interferometer is used to monitor the change in refractive index of a UV cured epoxy resin over a cure cycle. Fourier techniques are used to calculate the phase shift and frequency spectral amplitude of the associated fringe pattern during the cure. The results are compared with the refractive index change during cure calculated using a Fresnel reflection based technique.
\end{abstract}

Keywords: optical fibre, long period grating, cure sensor, Fresnel refractometer

\section{INTRODUCTION}

The on-line monitoring of the cure of epoxy resins using embedded optical fibre sensors has been reported previously ${ }^{1,2}$. Fibre optic methods offer clear advantages over traditional monitoring techniques because of their inherently small dimensions and high sensitivity. Spectroscopic methods predominantly use the fibre as a means for delivering the light to and from a sample ${ }^{1}$. However, direct interaction of the propagating mode with the surrounding environment has also been reported, achieved by etching or side polishing the optical fibre to remove the cladding and thus exposing the evanescent tail of the core mode field ${ }^{3}$.

Long period gratings (LPGs) are core to cladding mode coupling devices that allow analysis of the cladding mode properties whose evanescent tail interacts with the surrounding environment, without the need for removal of the cladding. This is particularly attractive for applications requiring embedding of the optical fibre, such as the monitoring of the cure of composite materials. LPGs consist of a periodic refractive index modulation of the core of an optical fibre, commonly formed by exposing the fibre to UV radiation. The refractive index modulation acts to couple energy from the propagating core mode to a discrete set of co-propagating cladding modes. The cladding modes attenuate rapidly, and the resultant transmission spectrum exhibits a series of attenuation bands.

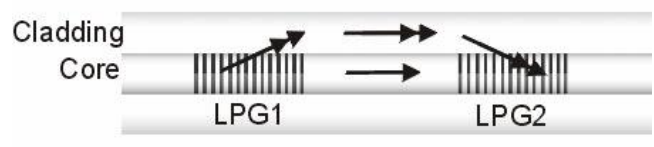

(a)

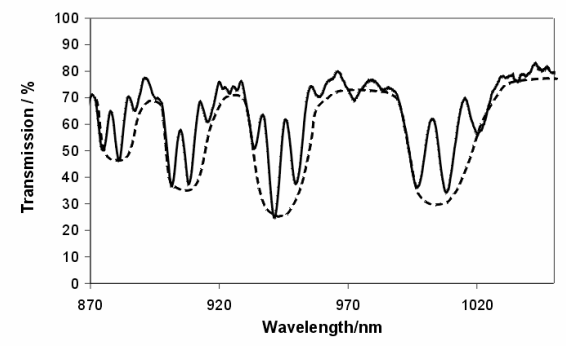

(b)

Fig 1. (a) Operation of cascaded LPGs as an MZI (b) channelled spectra in the attenuation bands [6]

When a pair of LPGs is written in series in an optical fibre they form an in-fibre Mach-Zehnder interferometer (MZI), as illustrated in Figure 1. The first LPG acts to couple a proportion of the propagating mode into the cladding. The cladding mode is re-coupled into the core by the second LPG, where it interferes with the core mode, introducing a sinusoidal channelled spectrum in the attenuation bands. The frequency of this channelled spectrum or fringe pattern in the spectrum of cascaded LPGs is determined by the separation of the LPGs. The phase is influenced by measurand-induced changes in the effective index of the cladding modes and the visibility is dependent upon factors such as the coupling

Third European Workshop on Optical Fibre Sensors, Antonello Cutolo, Brian Culshaw, José Miguel López-Higuera, Eds., Proceedings of SPIE Vol. 6619,66190V, (2007) $\cdot 0277-786 \mathrm{X} / 07 / \$ 18 \cdot$ doi: 10.1117/12.738364 
efficiency of the LPGs and by the cladding mode attenuation. Cascaded LPGs have been used to sense bending ${ }^{4}$, external index of refraction ${ }^{5,6,7}$, temperature and transverse load ${ }^{6}$. In this paper, an LPG based MZI is used to monitor the refractive index changes that occur during the cure of a UV cured epoxy resin (EpoTek OG-134).

\section{THEORY}

Assuming that the LPGs are identical, have a $3 \mathrm{~dB}$ coupling strength, and that the attenuation of the cladding modes can be neglected, the resulting intensity pattern can be described by equation 1 .

$$
I=I_{0}(\lambda)\left(1-\cos \left(\frac{2 \pi}{\lambda}\left(n_{\text {eff_co }}-n_{\text {eff_cl }}^{i}\right) L+\Phi\right)\right)
$$

where $I_{0}(\lambda)$ represents the spectral form of the attenuation band envelope, $n_{\text {eff_co }}$ and $n_{\text {eff_cl }}^{i}$ are the effective indices of the core and of the $i^{\text {th }}$ cladding mode respectively, $L$ is the fibre length separating the LPG pair and $\Phi$ is the phase imposed by the LPGs. The Fourier transform of such an intensity pattern is illustrated in Figure 2. One distinct peak is clearly visible on either side of the central DC peak, the amplitude of which is truncated in the interest of clarity.

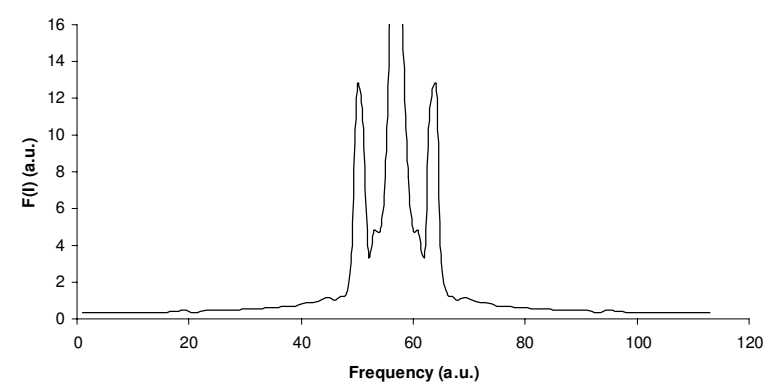

Fig 2. The Fourier transform of the intensity spectrum of an optical fibre containing a cascaded LPG interferometer.

The peaks represent the single frequency present in the induced fringe pattern, which is determined by the physical separation of the LPGs. A change in local environmental parameters results in a change in the effective refractive indices of the core and cladding modes. For small changes this appears as a change in the phase of the fringe pattern, which may be determined from analysis of the Fourier Transform of the transmission spectrum. The frequency was isolated and, after translation to the origin, the arctangent of the ratio of the real to imaginary components was used to determine the phase $^{6,7}$. The phase values obtained in this way were wrapped modulo $2 \pi$, resulting in discontinuities in the phase, which were then unwrapped.

\section{EXPERIMENT}

A Fresnel refractometer was used to provide an alternative measurement of the refractive index. The experimental configuration is shown in Figure 3. The output from a laser diode operating at $800 \mathrm{~nm}$ was intensity modulated at a frequency of $270 \mathrm{~Hz}$ and coupled into a network of $3 \mathrm{~dB}$ directional couplers. The Fresnel reflection from the fibre/resin interface was monitored using photodiode $\mathrm{pd}_{1}$. In the second arm, photodiode $\mathrm{pd}_{2}$ monitored the reflection from the air/fibre interface, acting as an intensity reference to aid the normalisation of the signal and to account for any change in power coupled into the optical fibre from the laser diode. The outputs from the photodiodes were monitored using lockin amplifiers. To minimize any contributions from unwanted Fresnel reflections from the unused port of the $3 \mathrm{~dB}$ coupler, micro bends were induced in the fibre to form a high attenuation return path for the reflections. The refractive index determined using the refractometer ${ }^{2,9}$ is given by equations $2-4$.

$$
\begin{gathered}
n=n_{\text {eff } \_ \text {co }}[(1-\zeta) /(1+\zeta)] \\
\zeta=\frac{n_{\text {eff_co }}-n_{a}}{n_{\text {eff_co }}+n_{a}} \frac{1}{\sqrt{R}} \\
R=\frac{V_{\text {air }}}{V_{\text {epoxy }}}
\end{gathered}
$$


where, $n_{a}$ is the refractive index of air, $V_{\text {air }}$ is the voltage produced by photodiode $\mathrm{pd}_{2}$, obtained for the reflection from the fibre/air interface and $V_{\text {epoxy }}$ is the voltage produced by photodiode $\mathrm{pd}_{1}$, corresponding to the reflection from the fibre/resin interface. The cascaded LPG pair was fabricated in a hydrogen loaded single mode optical fibre with a cut-off wavelength of $650 \mathrm{~nm}$ by UV exposure though a phase mask. The LPGs were each of length $20 \mathrm{~mm}$ with a period of $400 \mu \mathrm{m}$ and were separated by $40 \mathrm{~mm}$.

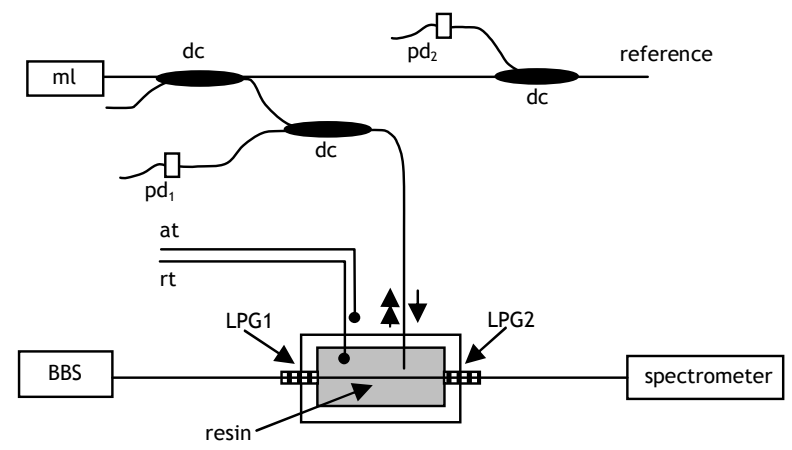

Fig 3. Experimental configuration. ml, modulated laser diode light source; pd, photodiode; dc, directional coupler;

BBS, broadband source; LPG, long period grating; rt, resin thermocouple; at, ambient thermocouple.

A UV cured epoxy resin, EpoTek OG134, with an uncured refractive index of 1.4163 measured at $589 \mathrm{~nm}^{10}$, was used as the test resin. A UV lamp with an output power of $4 \mathrm{~W}$ and with a spectral peak at $365 \mathrm{~nm}$ was positioned to provide a fluence of $6.57 \mathrm{~mW} / \mathrm{cm}^{2}$ in order to slow the cure reaction. Only the length of fibre separating the LPGs was immersed in the resin, as indicated in Figure 3. Thermocouples were placed in the resin and close to the sample to record resin and ambient temperature. The cascaded LPG spectrum and the Fresnel reflection were recorded at 6 second intervals during the cure. The Fourier transform of the optical spectrum was analysed to yield the phase change of each of the fringe patterns at a specified wavelength in the spectrum.

\section{RESULTS}

Figure 4 shows the calculated phase shift as the resin was cured over a 4 hour period in comparison with the refractive index measured by the Fresnel refractometer. The measured phase shift showed a positive trend which concurred with the increasing refractive change during cure. The origin of the features at 35 minutes and 55 minutes are associated with small temperature fluctuations, as can be seen in Figures 6(a) and (b), where the temperature of the resin, monitored using a thermocouple, and the phase change are plotted on the same graph. As the refractive index of the resin approaches that of the cladding, the sensitivity of the cladding mode effective index increases. The features in Figure 6 correspond to thermally induced refractive index changes. A small decrease in temperature corresponds to a small increase in refractive index due to the thermo-optic effect which induces a positive phase shift. The increased sensitivity is accompanied by an increase in the attenuation of the cladding modes, and an associated decrease in visibility, as shown in Figure 5.

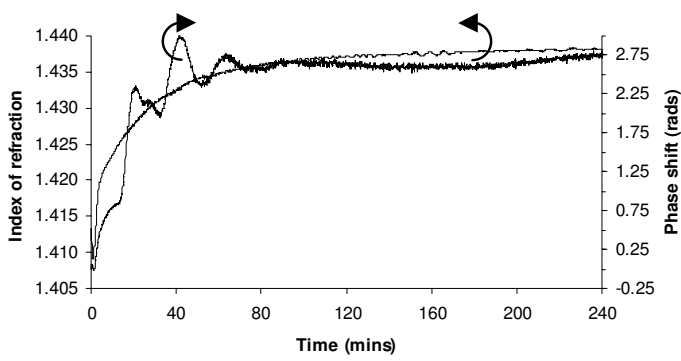

Fig 4. Phase shift of channelled spectrum (dashed) and refractive index (solid) change during epoxy cure.

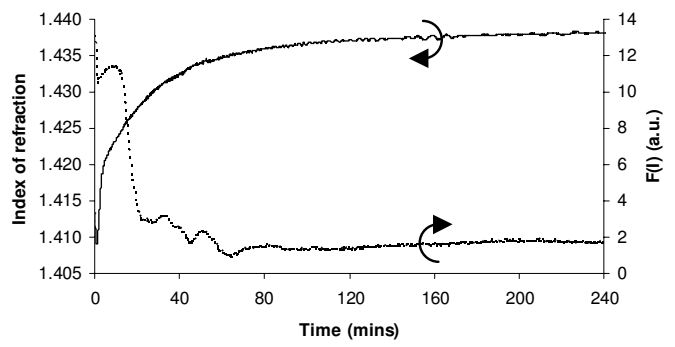

Fig 5. Frequency amplitude, F(I) (dashed) and refractive index (solid) change during cure. 
Figure 6 illustrates the potential for the use of an LPG MZI as a highly sensitive cure sensor. The exothermic reaction which occurred shortly after the resin was illuminated by the UV lamp is clearly identified. The high sensitivity of the system, exhibited as the refractive index of the resin approaches that of the cladding, may be useful for identifying the occurrence of exothermic or endothermic reactions within a complex component for monitoring systems where tight control of the temperature is required. It is interesting to note that, despite the reduction in the visibility of the channelled spectrum, it is still possible to measure the change in phase. Previous work ${ }^{2}$ has shown that, when using an LPG as a refractometer to monitor the same system, the corresponding reduction in the depth of the attenuation band prevents the measurement of the refractive index induced wavelength shift.

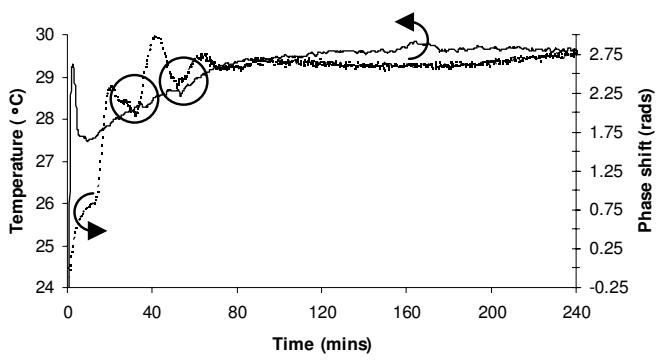

(a)

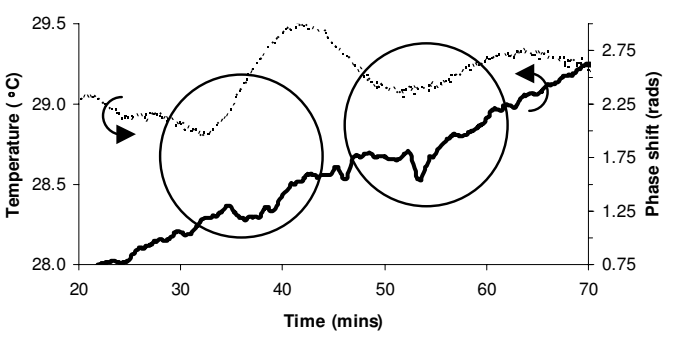

(b)

Fig 6. (a) Phase shift (dashed) of sinusoidal channeled spectrum in band 5 of LPG based MZI with temperature response (solid) of EpoTek OG-134 resin during cure. (b) Closer detail of the temperature response, grey line shows ambient temperature.

\section{SUMMARY}

A cascaded LPG MZI has been used to monitor the cure of an epoxy resin. The phase shift and frequency amplitude of the associated interferometric fringes were calculated using Fourier analysis and compared with the calculated refractive index change of a Fresnel based refractometer during the cure of an epoxy resin. The results compare favourably and indicate the possibility of a highly sensitive cure sensor. The authors gratefully acknowledge the support for this work from the Engineering and Physical Sciences Research Council (EPSRC), UK.

\section{REFERENCES}

1. B. Degamber and G. F. Fernando, "Process monitoring of fiber-reinforced polymer composites," MRS Bulletin 27, 370-80 (2002).

2. S. J. Buggy, E. Chehura, S. W. James and R. P. Tatam, "Fibre Gratings for Cure Monitoring of Epoxy Resins," OFS - 18, (2006).

3. T. Takeo and H. Hattori, "Optical Fiber Sensor for Measuring Refractive Index," Japan J. Appl. Phys 21, 1509 $1512(1982)$

4. B. H. Lee and J. Nishii, "Bending sensitivity of in-series long-period gratings," Opt. Lett. 23, 1624 - 1626 (1998).

5. O. Duhem, J.F. Henninot and M. Douay, "Study of in fiber Mach-Zehnder interferometer based on two spaced 3-dB long period gratings surrounded by a refractive index higher than that of silica," Opt. Commun. 180, 255 - 262 (2000).

6. Y.G. Han, B.H. Lee, W.T. Han, U.C. Paek and Y.Chung, "Fibre-optic sensing applications of a pair of long-period fibre gratings," Meas.Sci.Technol. 12, 778 - 781 (2001).

7. R. P. Murphy, S. W. James and R. P. Tatam, "Multiplexing of fibre optic long period grating based interferometric sensors", J. Lightwave Technol. in press, (2007).

8. M. Takeda, H. Ina, S. Kobayashi, "Fourier-transform method of fringe-pattern analysis for computer-based topography and interferometry," J. Opt. Soc. Am. 72, 156 - 159 (1982).

9. C. B. Kim and C. B. Su, "Measurement of the refractive index of liquids at 1.3 and 1.5 micron using a fibre optic Fresnel ratio meter," Meas.Sci.Technol. 15, 1683 - 1686 (2004).

10. Material safety data sheet Epo-Tek OG134, Epoxy Technology, Billerica, USA, www.epotek.com 